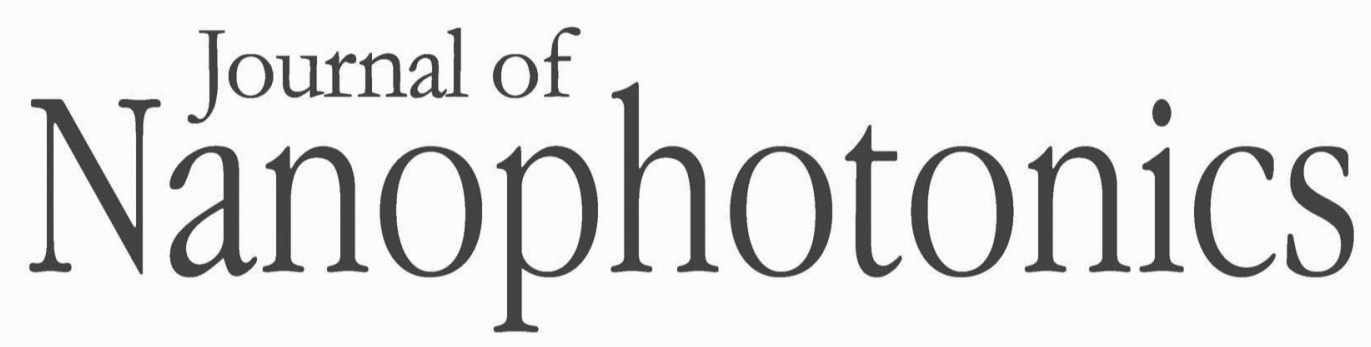

\title{
Bilayer hybrid nanoimprinting method for fabricating embedded silver nanostructure arrays with enhanced photoluminescence
}

Jun-Hyuk Choi

Sang-Keun Sung

Chul-Hyun Kim

Yeon-Ho Jung

Joo-Yun Jung

Jun-Ho Jeong

Eung-Sug Lee

\section{O SPIE}




\title{
Bilayer hybrid nanoimprinting method for fabricating embedded silver nanostructure arrays with enhanced photoluminescence
}

\author{
Jun-Hyuk Choi,* Sang-Keun Sung, Chul-Hyun Kim, Yeon-Ho Jung, \\ Joo-Yun Jung, Jun-Ho Jeong, and Eung-Sug Lee \\ Korea Institute of Machinery and Materials, Daejeon 305-343, Republic of Korea
}

\begin{abstract}
A bilayer hybrid nanoimprinting (NI) method was developed for fabricating embedded metal nanopatterns with greater processability and improved reliability for enhanced photoluminescence (PL) in optoelectronic devices. Bilayer hybrid NI consists of the following: (a) spin-coating ultraviolet (UV) and thermally curable NI resists in sequence, (b) high-pressure thermal NI and UV exposure while maintaining the stamp in a pressed position, and (c) silver (Ag) deposition and lift-off using a thermal NI resist on the upper surface to create embedded Ag nanoarrays. Reference samples with no Ag nanopatterns and with protruding Ag dot-shaped nanopatterns were also fabricated for comparison. The transmittance and PL of all samples were measured. All samples containing Ag nanopatterns exhibited improved PL compared with reference samples with no Ag. For all pattern sizes, the samples with the embedded Ag nanoarrays exhibited the highest PL; the relative PL enhancements compared with samples with Ag dot-shaped nanoarrays were $32.2 \%, 36.1 \%$, and $62.7 \%$ for pattern sizes of 150, 200, and 265 nm, respectively. (C) The Authors. Published by SPIE under a Creative Commons Attribution 3.0 Unported License. Distribution or reproduction of this work in whole or in part requires full attribution of the original publication, including its DOI. [DOI: 10.1117/1.JNP.8.083089]
\end{abstract}

Keywords: bilayer resist; hybrid nanoimprinting; plasmonic field; photoluminescence.

Paper 13115 received Oct. 15, 2013; revised manuscript received Feb. 5, 2014; accepted for publication Feb. 10, 2014; published online Apr. 28, 2014.

\section{Introduction}

Metallic nanostructures, often referred to as metallic photonic crystals or plasmonic nanostructrures, can act as light-scattering centers through plasmonic resonant excitation and outcoupling of the incident electromagnetic field. The use of metallic nanostructures is known to improve the coupled emissions and energy transformation efficiencies in optoelectronic devices ${ }^{1-4}$ and the quality of sensing in sensors. ${ }^{5-7} \mathrm{New}$ advanced nanofabrication methods have recently enabled a wide range of other applications, including metallic conductor-based electronic devices ${ }^{8,9}$ and metal slits in optical modulators. ${ }^{10-12}$ A protruding metal nanodot configuration has been typically achieved through high-precision microfabrication tools for lift-off ${ }^{13-15}$ and direct-ion milling $^{16,17}$ of predefined patterns fabricated using nanoimprinting (NI) or soft nanolithography. Corrugated metal nanostructures have also been considered instead of isolated metal dots for use in optoelectronic devices. ${ }^{2-4,18}$ However, both sputtered and evaporated metal films exhibit inherent surface roughness, and their use leads to problems such as current leakages and resistivity increases.

Corrugated dielectric nanostructures have been used to improve emission efficiency via enhanced out-coupling of trapped photons in the waveguide mode. ${ }^{19-22}$ However, these efficiency improvements are achieved at the cost of increased leakage currents because conformally deposited electrodes and emitters are characterized by elongated current paths and degraded uniformity. For example, nanopatterned organic light-emitting diodes (OLEDs) with a photonic crystal structure exhibit even lower luminous efficiencies at current densities larger than

*Address all correspondence to: Jun-Hyuk Choi, E-mail: junhyuk@kimm.re.kr 
$5 \mathrm{~mA} / \mathrm{cm}^{2}$; however, the efficiencies can be increased by $13 \%$ by planarization of the device with multiple spin-coated layers of $\mathrm{ZnO}$ sol-gel. ${ }^{21}$ Alternatively, to reduce current leakage and enhance light extraction, multiple spin-coated layers and physical pressing of a higher refractive sol-gel oxide, $\mathrm{TiO}_{2}$, can be used to fill in and smooth any "pillar"-like nanostructures on the glass substrate prior to deposition of the anode in OLED fabrication. ${ }^{22}$ In fact, if corrugated Ag nanostructures exceed a critical height, then the increase in efficiency attributable to the Ag nanostructures is offset by efficiency decreases attributable to a grating effect on the electroluminescence intensity and absorption. ${ }^{2}$ Thus, the corrugated nanopattern-endowed benefits must be balanced against effects such as light scattering and spectral absorptions.

Similar effects have been observed when plasmonic metal structures have been incorporated into devices. Relevant previous studies have attempted to selectively infiltrate metal nanoparticles directly into nanopattern holes ${ }^{23,24}$ or have used a template stripping method. ${ }^{25-28}$ For example, the embedding of gold $\mathrm{Au}$ ) nanowires into an indium tin oxide anode layer was achieved by spin coating and surface energy-driven confinement of Au nanoparticles into the grating grooves. ${ }^{23}$ In this case, strong coupling between the waveguide mode and the plasmon resonance nanowire in the transverse magnetic field mode could enhance direct-current conductivity and conferred unique optical behaviors. Furthermore, the template stripping method, initially developed by Frey et al. ${ }^{25,26}$ has been shown to generate the same level of surface roughness of the embedded metal nanopattern surface as the surface of the target template for metal deposition. Accordingly, the corrugation levels of embedded metal nanopatterns should be optimized to achieve plasmonic resonance improvements in devices.

Prior studies as referred above have significantly contributed to the understanding of the implications of using embedded metal nanostructures. However, in most cases, fabrication approaches have been complicated, requiring numerous process steps and leading to a lack of processability and reliability. In this study, a cost-effective and simple fabrication strategy for embedding metal nanoarrays was developed. Our method is based on bilayer hybrid NI [sequential thermal and ultraviolet (UV) NI in a single process step] followed by accessible metal lift-off. Transmittance spectra and photoluminescence (PL) enhancements observed in devices fabricated using our method were examined terms of the embedded nanopattern size in comparison with corresponding metal nanodot configurations.

\section{Experiments}

As shown in Figs. 1(a) and 1(b), the first step of fabrication is coating with UV-curable and thermally curable NI resists (UV cur 21, mr-I 7010E, MRT Inc., Korea). The UV resist was partially cured prior to deposition of the thermal resist to ensure that it would not be removed upon spin coating of the thermal resist. The UV resist was sufficiently precured to maintain its integrity while also achieving the minimal viscoelastic properties required for molding during the subsequent high-pressure imprinting. Because the UV and thermal NI resists had compatible chemistry and similar solvents, they tended to mix without precuring of the UV resist. Either UV exposure in an oxygen-free environment or vacuum plasma could be used for the precuring.

Next, hard transparent stamps with protruding pillar arrays, which were replicated from $\mathrm{Si}$ master patterns, were applied in the NI step, as shown in Fig. 1(c). Three types of stamps were applied: a hexagonal array with 150-nm diameter and 300-nm-high nanopillars, a hexagonal array with 200-nm diameter and 250-nm-high nanopillars; and a hexagonal array with 265-nm diameter and 270-nm-high nanopillars. Their pattern periodicity was identically twice of their diameters. The given pattern sizes were chosen to minimize the nonradiative PL decay. ${ }^{27}$ Bilayered resists were imprinted under increasing pressures up to $\sim 50$ bar at $150^{\circ} \mathrm{C}$ for $20 \mathrm{~min}$ in a vacuum chamber, and then subsequently exposed to a UV lamp (energy intensity $\sim 35.5 \mathrm{~mW} / \mathrm{cm}^{2}$ ) for 3 min while imprinted with the stamp. The quality of the pattern transferred to the underlying UV-curable resist was evaluated after mold release and removal of upper thermal resist layer. The thickness of the thermal resist was optimized to more effectively transfer the pattern to the underlying UV resist layer; the optimal thickness for the thermal resist layer was found to be $<100 \mathrm{~nm}$, which could be achieved through adjustments in dilution, spin speed, and spin time. A 25- to 30-nm layer of Ag was then evaporated onto the device using an 


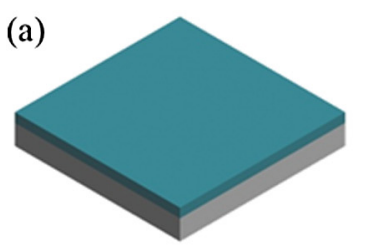

(b)

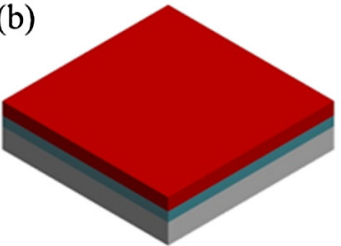

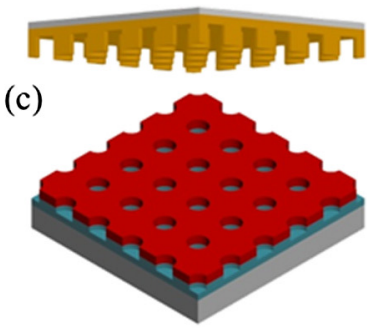

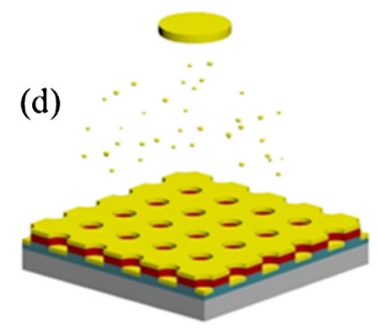

(e)

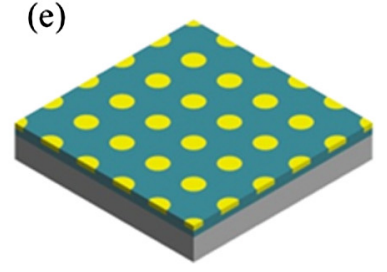

Fig. 1 Schematic diagram of the bilayer hybrid nanoimprint (NI) process for fabricating embedded metal nanopatterns. The process consists of (a) coating the substrate with an ultraviolet (UV)-curable resist, (b) coating the substrate with a thermal $\mathrm{NI}$ resist, (c) thermal $\mathrm{NI}$ followed by UV exposure, (d) evaporation of $\mathrm{Ag}$, and (e) lift-off of $\mathrm{Ag}$ metal and thermal $\mathrm{NI}$ resist.

in-house electron-beam evaporator, as shown in Fig. 1(d), to fill the holes on the bottom side of the UV-cured resist layer. The Ag deposited on the tops and sidewalls of the imprinted holes on the upper thermal resist was then easily removed along with the thermal resist in acetone under ultrasonication for $5 \mathrm{~min}$, as shown in Fig. 1(e). Thus, Ag remained only in the imprinted holes on the UV-cured resist, thereby completing the embedded Ag nanopattern configuration. Reference samples for comparison were made with protruding dot-shaped Ag nanopatterns. These counterpart samples were fabricated by Ag lift-off on the predefined NI patterns using lift-off resist and a Si-based etch mask material. ${ }^{15}$

The transmittance spectra of the embedded $\mathrm{Ag}$ and protruding $\mathrm{Ag}$ nanodot samples were measured in the UV-visible wavelength range (NEOSYS-2000, SCINCSO Inc., Korea). Prior to the deposition of PL layer, a 20-nm oxide layer was deposited as a spacer on both the protruding Ag dot-shaped and embedded Ag samples to prevent quenching to eliminate PL quenching. Pre-existing native oxide should add, which was considered to be still in the optimum range (20 to $25 \mathrm{~nm}$ ) of quenching-resistant thickness of oxide layer. ${ }^{29}$

The PL materials used were green-emitting tris[2-(p-tolyl)pyridine] iridium (III) [ $\left.\operatorname{Ir}(\mathrm{mppy})_{2}\right]$ (EM Index Co., Seoul, Korea). The PL materials were dissolved in chlorobenzene, doped with polymethymethacrylate (PMMA), and spin-coated onto the oxide layer on the sample substrates. It was baked on a hotplate at $80^{\circ} \mathrm{C}$ for $20 \mathrm{~min}$. The PL spectrum of $\operatorname{Ir}(\mathrm{ppy})_{3}$ complexes-doped PMMA films showed the excitation peak at 370-nm wavelength. The PL spectra were measured using a Hitachi F-4500 fluorescence (Tokyo, Japan) spectrophotometer focused at green emission to compare the different $\mathrm{Ag}$ configurations and to investigate the size dependence in combination with the transmittance spectra. The solid-state emission measurements were carried out by using each film supported on a quartz substrate that was mounted with front-face excitation at an angle of $<45 \mathrm{deg}$. Each emitting film was excited with several portions of visible light from a xenon lamp.

\section{Results and Discussion}

Scanning electron microscopy (SEM) was used to obtain images of the fabrication process. The SEM images of the embedded Ag nanoarrays presented in Figs. 2(a) and 2(b) correspond to the process step shown in Fig. 1(c), and the Ag deposition and lift-off step depicted in Fig. 1(d), respectively. As shown in Fig. 2(b), and as a result of the resist thickness optimization experiments, high-pressure NI on the top surface of the UV curable resist formed imprinted holes $\sim 30 \mathrm{~nm}$ in depth on the underlying thermal resist. This depth was sufficient for embedding the evaporated $\mathrm{Ag}$ in the subsequent process step. The SEM images of the embedded $\mathrm{Ag}$ 

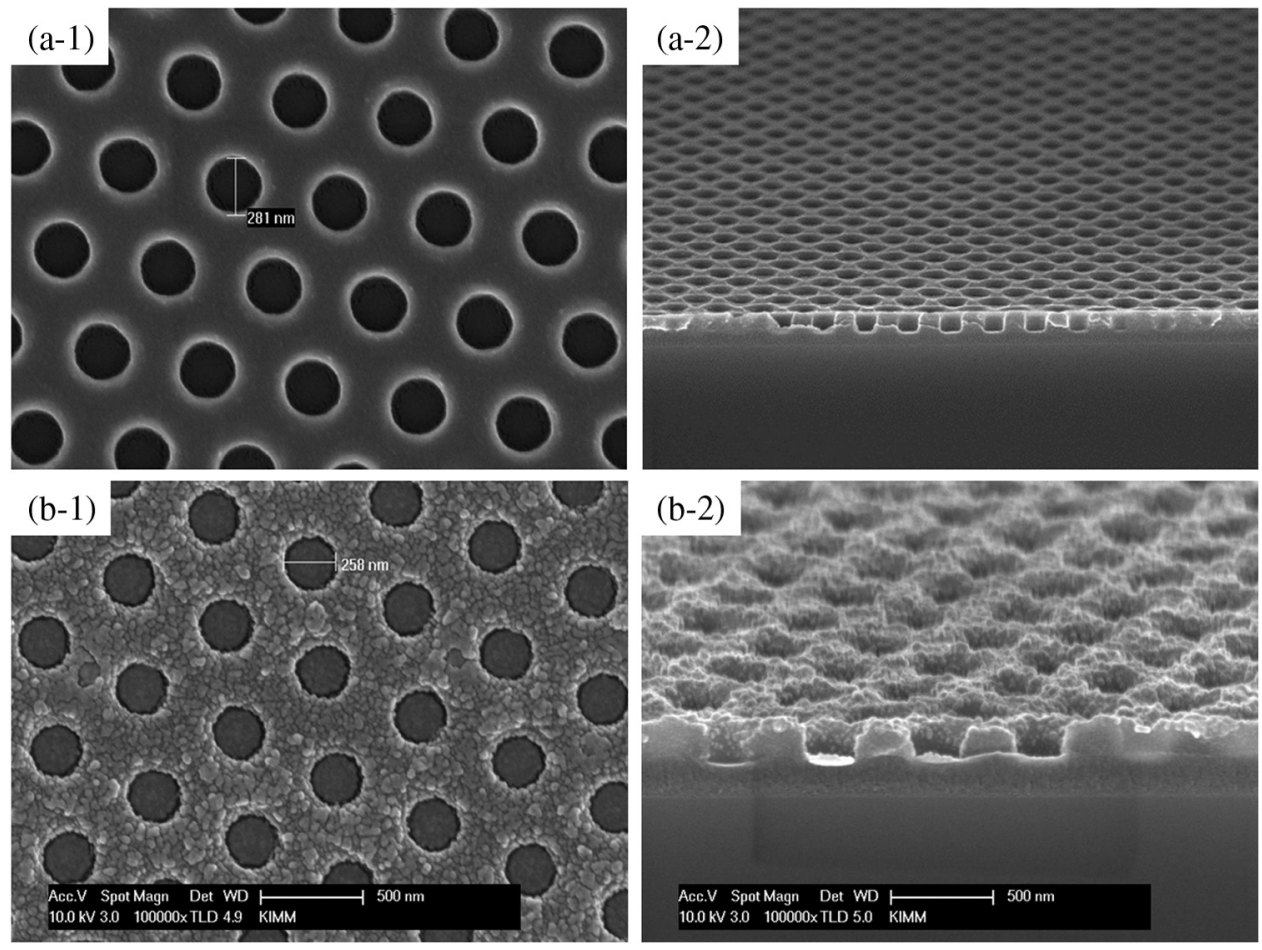

Fig. 2 Scanning electron microscopy (SEM) images of the bilayer hybrid NI process corresponding to (a) the thermal $\mathrm{NI}$ and UV exposure step depicted in Figs. 1(c) and 1(b) the Ag evaporation step depicted in Fig. 1(d).
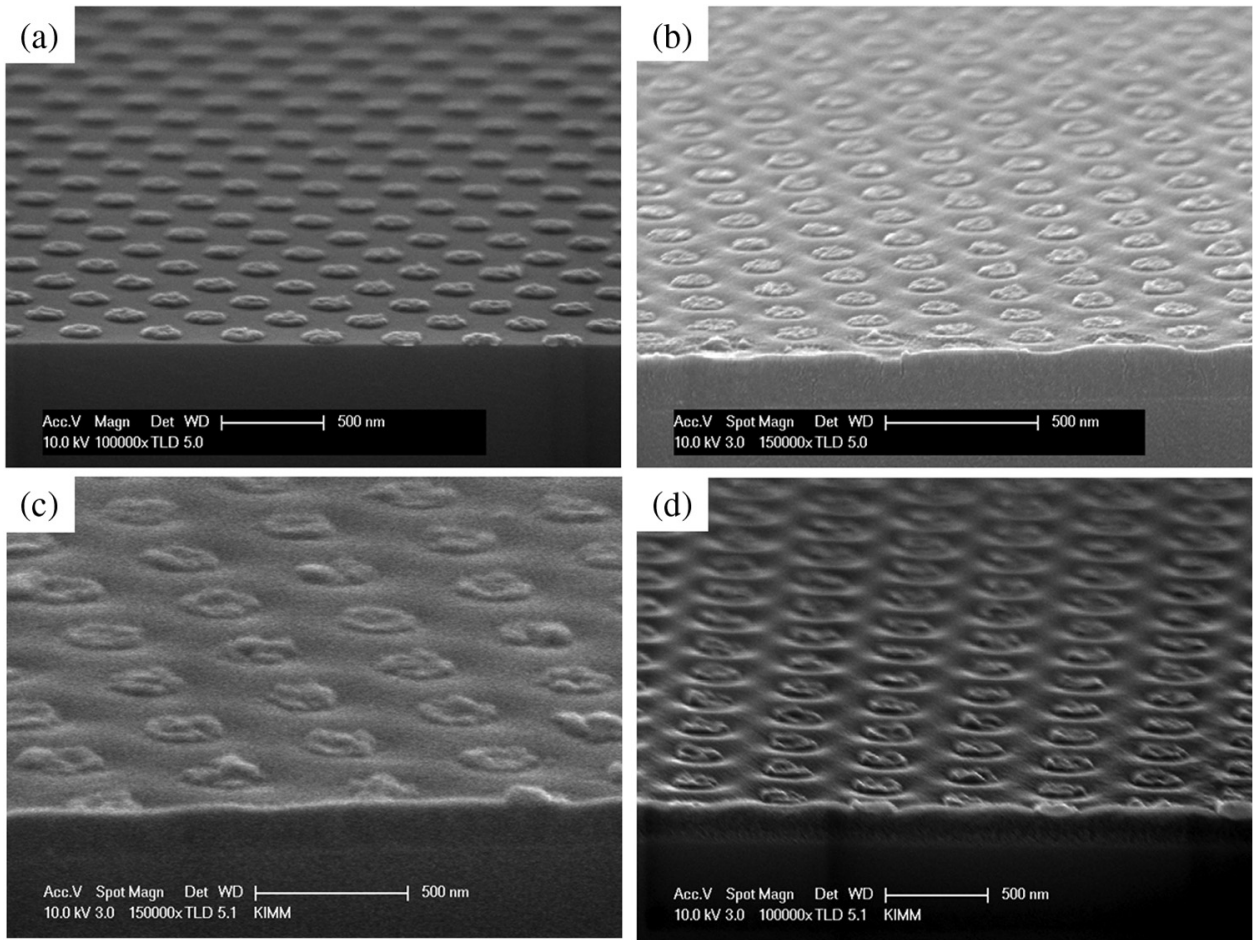

Fig. 3 The SEM images of Ag hexagonal nanoarrays (a) protruding dot-shaped Ag nanopattern with 200-nm-diameter structures, and (b)-(d) the embedded configuration with 150-, 200-, and 265-nm-diameter structures, respectively. 
nanoarrays fabricated for three hole diameters $(150,200$, and $265 \mathrm{~nm})$ are presented in Figs. 3(b)-3(d). The SEM image of a 200-nm-diameter protruding dot-shaped Ag nanoarray, which was fabricated using metal lift-off assisted with a Si-based etch mask material and lift-off resist to minimize the rabbit-ear side profile for the comparative study, is shown in Fig. 3(a).

Figures 4(a) and 4(b) present atomic force microscopy (AFM) images of the Ag nanoarrays with feature diameters greater than $150 \mathrm{~nm}$. Figures 4(a) and 4(b) correspond to the nanoarrays depicted in Figs. 3(c) and 3(d) above, respectively. An AFM image of the 150-nm nanoarray [corresponding to the SEM image in Fig. 3(b)] is excluded because inaccurate measurement deviations associated with the AFM tip size were observed for this specimen. The AFM images for the 200- and 265-nm nanoarrays were qualitatively well matched to the SEM images. The structures exhibited clear but slightly exaggerated profiles with pronounced edge waviness around the imprinted holes. The stretched waviness of the imprinted hole surfaces on the UV-cured resist and the ball-shaped silver dots inside the holes are expected to reduce the corrugation period roughly by half, so-called the reduced pseudo-periodicity resulting from the deformed pattern profile. In addition, the features in the embedded Ag nanoarrays were projected more distinctly in the AFM images than in the SEM images because of scanning inaccuracy and interface tolerances.

The different Ag nanoarray configurations resulted in different optical performance. Figure 5 shows the dependence of transmittance on the feature size for two given Ag surface configurations. The transmittance fluctuated more in the protruding $\mathrm{Ag}$ nanodot-shaped samples [Fig. 5(a)] than in the bilayer-imprinted embedded Ag nanoarray samples [Fig. 5(b)]. For both shapes, the plasmonic peak wavelength was red shifted as the feature size increased. It moved from 700 to $1050 \mathrm{~nm}$ in wavelength as the dot size increased from 150 to $265 \mathrm{~nm}$. Transmittances at the plasmonic extinction peak wavelength were greater for the embedded
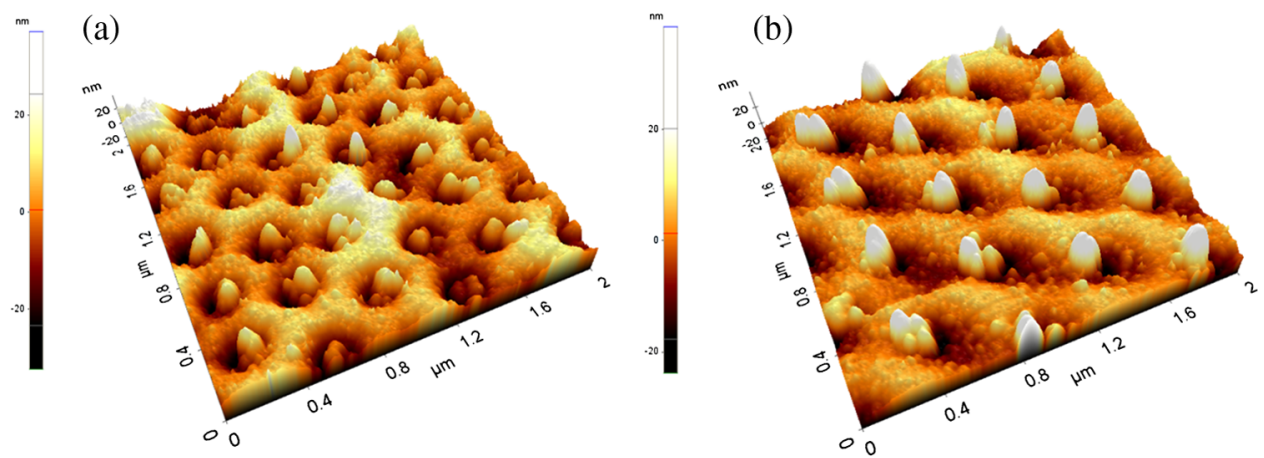

Fig. 4 Atomic force microscopy profile images of embedded Ag nanoarrays with (a) 200- and (b) 265-nm-diameter structures.
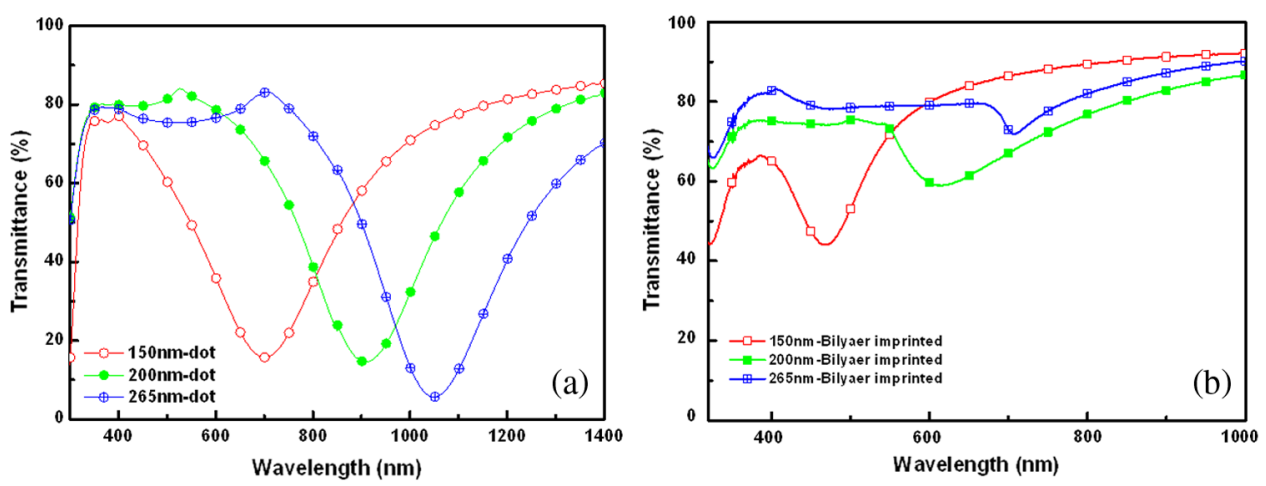

Fig. 5 Transmittance spectra of Ag nanoarrays with 150-, 200-, and 265-nm configurations: (a) dot-shaped protruding Ag nanoarrays and (b) embedded Ag nanoarrays fabricated using bilayer hybrid NI. 
Ag nanoarray than for the Ag dot-shaped nanoarray; the transmittance values were $15.7 \%$ and 5.7\% for the Ag dot-shaped nanoarray with 150- and 265-nm features, respectively, and $44.1 \%$ and $72 \%$ for the embedded Ag nanoarray with 150- and 265-nm features, respectively. Blue shifts of the plasmonic extinction peak wavelength were observed in all of the embedded Ag nanoarray samples for all pattern sizes. The blue shifts were attributed to the somewhat wavy edge profiles between the imprinted holes and the silver inclusions, different materials between the silver patterns, and more effective higher order diffractions that were not picked up by our measurement scheme. However, scattering effects, particularly back-scattering, seem to dominate the plasmonic extinctions obtained for the dot-shaped configurations [Fig. 5(a)]; thus, it was conversely inferred that the decreased transmittance observed in the embedded Ag configuration could be attributed to plasmonic absorptions [Fig. 5(b)].

The PL measurements as a function of configuration and pattern size are summarized in Fig. 6. The structural configuration remarkably influenced the PL. For green-emitting PL materials, the enhancement factor increased with the pattern size for the embedded Ag nanopattern samples [Fig. 6(a)], although slight spectral mismatches still existed. Compared with the reference sample with no $\mathrm{Ag}$, the PL enhancements at an emitting wavelength of $510 \mathrm{~nm}$ were $38.6 \%, 55.4 \%$, and $88.0 \%$ for nanoarrays with 150-, 200-, and 265-nm features, respectively. The embedded Ag nanoarray configurations exhibited even larger PL enhancements than the dot-shaped Ag nanoarrays for all pattern sizes, as shown in Figs. 6(b)-6(d). The 150-nm dot-shaped Ag nanoarray resulted in a $4.8 \%$ enhancement compared with the reference sample with no Ag, whereas its embedded $\mathrm{Ag}$ counterpart exhibited an enhancement of $38.6 \%$, which corresponds to a relative PL enhancement over the dot-shaped Ag nanoarray of $\sim 32.2 \%$. The PL enhancements of the dot-shaped Ag nanoarrays were $36.1 \%$ and $62.7 \%$ for the pattern sizes of $200 \mathrm{~nm}$ and $265 \mathrm{~m}$, respectively, which even increased up to $55.4 \%$ and $88.0 \%$ for their counterpart embedded $\mathrm{Ag}$ configurations with the relative PL enhancement ratios of $14.2 \%$ and $15.6 \%$, respectively.
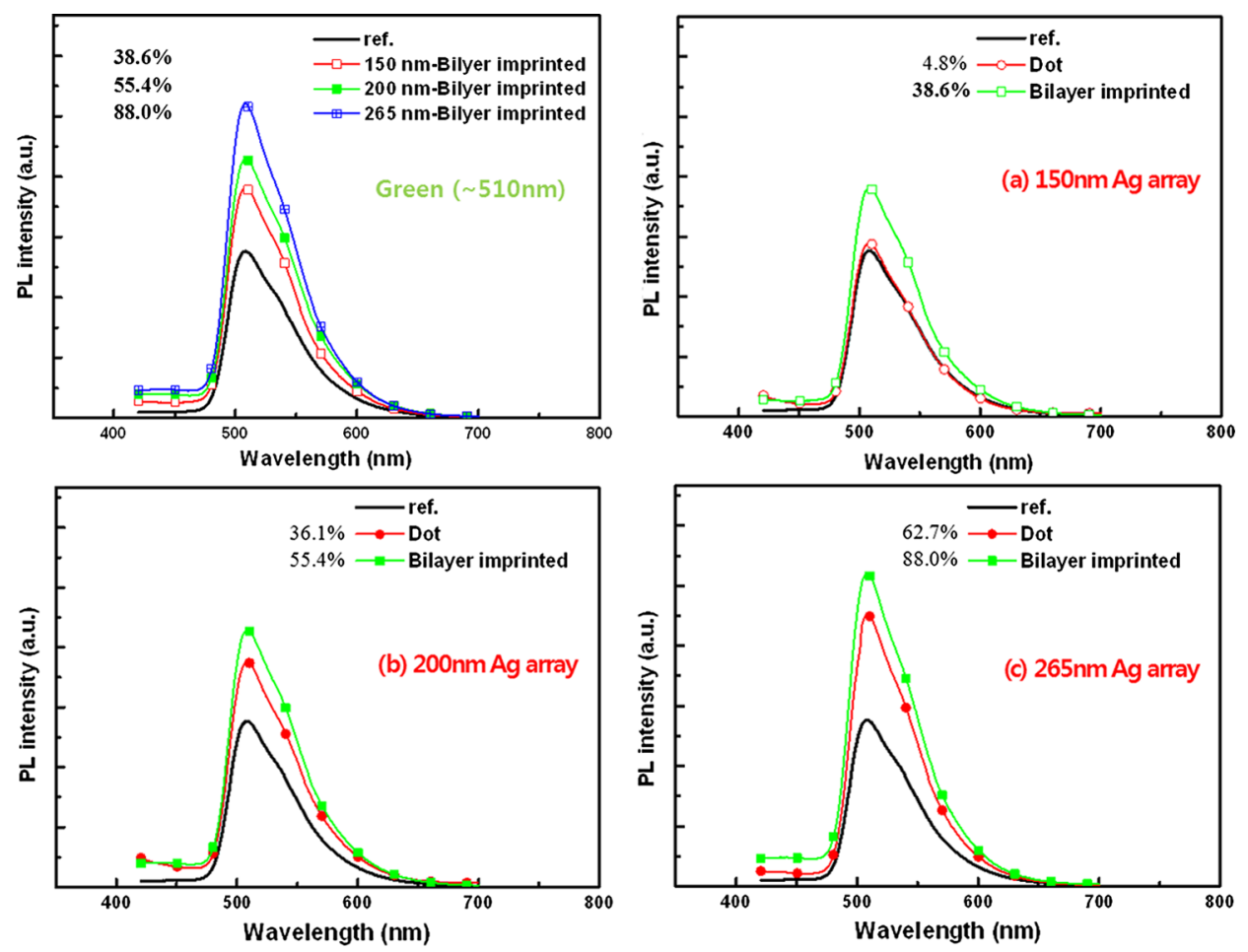

Fig. 6 Photoluminescence $(\mathrm{PL})$ enhancements with green-emitting PL materials $\left[\mathrm{Ir}(\mathrm{mppy})_{3}\right]$ on SiOx-deposited silver-nanopatterned glass subtrates: (a) the dependence of PL on the size of the embedded Ag nanoarray, and (b)-(d) comparisons of protruding dot-shaped Ag nanoarrays (200-nm diameter) and embedded Ag nanoarrays with diameters of 150, 200, and $265 \mathrm{~nm}$, respectively. 
The larger PL enhancements for the Ag-embedded nanoarrays could be partially attributed to decreased scattering loss observed in the transmittance measurements (Fig. 5), which might result in a plasmonic field-driven increase in photon absorption in the PL layer. However, the observed transmittance differences between the embedded and the dot shapes are trivial for all the given pattern sizes in PL at the excitation wavelength of $510 \mathrm{~nm}$. Hence, this would not fully explain the much higher relative PL enhancements of the embedded Ag nanoarrays. Thus, other phenomena must have also occurred. Higher order diffractive scattering would have also caused more photons to be absorbed in the PL layer, which would have contributed to an increase in PL for the embedded Ag nanoarrays compared with their counterpart dot-shaped Ag nanoarrays, but this phenomenon was not detected in the transmittance measurements. Instead, the blue shifted extinction peak wavelength may have more directly influenced the relative PL improvements, facilitating the plasmonic resonance matching condition and subsequently its near-field coupling to PL. At near the PL emitting wavelength, the surface plasmonic field energy can be resonantly out-coupled to facilitate PL emissions through repetitive charge generations and photon absorptions caused by the field intensification, ${ }^{30,31}$ and also the embedded Ag nanopatterns may have scattered more of the near-field luminescence that would have otherwise been trapped in the waveguide mode.

The increased photon absorptions and near-field plasmonic coupling efficiency would be the major causes of the larger PL improvements for the embedded Ag configurations. The decreased scattering loss, increased higher order diffractions, and blue shifting of the peak wavelength were the most likely underlying related factors leading to the PL improvements. These effects were more pronounced when Ag nanoarrays were embedded instead of protruding. The present study was focused on the development of a new fabrication strategy for embedded Ag nanopatterns and the exploration of their enhancement of plasmonic PL. Additional fundamental studies aimed at better understanding the enhancement physics are suggested.

\section{Conclusion}

A bilayer hybrid NI method followed by silver lift-off was developed for the fabrication of embedded Ag nanoarrays. In addition, the improved plasmonic-coupling effectiveness of embedded Ag nanoarrays was investigated through PL measurements in comparison with reference samples with no silver and their counterpart protruding dot-shaped Ag nanoarray configurations. The process was characterized by imprinting of the bilayer-deposited resist system (including UV-curable and thermally curable resists), leaving the imprinted holes on the underlying UV resist available for subsequent Ag deposition. The top surface of the thermal resist acted as a buffer layer for imprint pattern transfer to the underlying UV-curable resist and as a sacrificial layer for subsequent metal lift-off. As an additional benefit, the Ag trapped in the imprinted holes was more resistive to a swirling lift-off process using a wet chemical bath than to conventional lift-off.

Transmittance and PL were measured for the embedded Ag nanoarrays and protruding Ag dot-shaped nanoarrays with three different feature diameters as well as for reference samples with no Ag. The plasmonic peak wavelength was blue shifted, and the scattering loss was lower for the embedded Ag configuration than for the protruding Ag dot-shaped configuration. PL enhancements compared with reference samples were observed for all samples containing Ag nanoarrays; the observed enhancements were greater for the embedded Ag nanoarrays than for the Ag dot-shaped nanoarrays. Relative PL enhancements of embedded Ag nanoarrays compared with Ag dot-shaped nanoarrays were 32.2\%, 14.2\%, and 15.6\% for 150-, 200-, and 265-nm pattern sizes, respectively. The largest relative PL enhancement for $150 \mathrm{~nm}$ can be explained in a point of the spectral matching between the plasmonic extinction and the emitting wavelength of the given green PL material. The gap between plasmonic extinction wavelength and the emitting wavelength $(510 \mathrm{~nm})$ seems to be quite critical. It is smaller in the embedded ones than in the dot-shaped ones. It reduced from 190 to $40 \mathrm{~nm}$ in the diameter of $150 \mathrm{~nm}$, from 910 to $110 \mathrm{~nm}$ for $200 \mathrm{~nm}$, and from 1050 to $710 \mathrm{~nm}$ for $265 \mathrm{~nm}$. Hence, its highest rate (78.9\%) occurred for 150-nm pattern size, which probably leaded to the largest relative PL enhancement. The effects of the metal nanostructure size on PL intensity have been investigated in Ref. 32. 
The PL enhancements were evaluated using transmittance spectra and discussed from the view point of effective plasmonic near-field coupling efficiency and scatter-driven photon absorption in PL layer. The embedded configuration was the most effective for extracting the PL excitations and reducing the scattering loss. This study focused on presenting a new fabrication method for fabrication of embedded $\mathrm{Ag}$ nanopatterns and exploration of their enhancement of plasmonic PL. The presented bilayer hybrid NI method is simple and minimized the process steps required for fabricating embedded Ag nanopatterns.

\section{Acknowledgments}

This research was supported by grants from the Basic Science Research Program (20110028585) and the Global Frontier R\&D program (2011-0031563), funded by the National Research Foundation of Korea (NRF) under the Ministry of Education, Science, and Technology. Dr. Jun-Hyuk Choi developed and planned the present approach, and contributed to writing the paper. Mr Sang-Keun Sung contributed to metal evaporation and lift-offs. $\mathrm{Mr}$ Chul-Hyun performed the fabrication of nanopatterns by hybrid nanoimprint. Mr Yeon-Ho Jeong contributed to measuring photoluminescence and transmittance spectra. Dr. Joo-Yeon Jeong designed mold patterns by simulations. Dr. Jun-Ho Jeong took responsibility for nanoimprint mold fabrications. Dr. Eung-sug Lee contributed to preparing all material choices and provided all valuable suggestions in the nanoimprint.

\section{References}

1. M. G. Kang et al., "Efficiency enhancement of organic solar cells using transparent plasmonic Ag nanowire electrode," Adv. Mater. 22(39), 4378-4383 (2010), http://dx.doi.org/10 .1002/adma.v22:39.

2. X. L. Zhang et al., "Grating amplitude effect on electroluminescence enhancement of corrugated organic light-emitting devices," Appl. Phys. Lett. 36(19), 3915-3917 (2011), http://dx.doi.org/10.1364/OL.36.003915.

3. J. Feng and T. Okamoto, "Enhancement of electroluminescence through a two-dimensional corrugated metal film by grating-induced surface-plasmon cross coupling," Opt. Exp. 30(17), 2302-2304 (2005), http://dx.doi.org/10.1364/OL.30.002302.

4. V. Reboud et al., "Nanoimprinted plasmonic crystals for light extraction application," Microelectron. Eng. 87(5), 1367-1369 (2010), http://dx.doi.org/10.1016/j.mee.2009.12 .030 .

5. J. Maria et al., "Optimization of 3D plasmonic crystal structures for refractive index sensing," J. Phys. Chem. C 113(24), 10493-10499 (2009), http://dx.doi.org/10.1021/ jp9024552.

6. J. Yao et al., "Seeing molecules by eye: surface plasmon resonance imaging at visible wavelengths with high spatial resolution and submonolayer sensitivity," Angew. Chem. Inst. Ed. 120(27), 5091-5095 (2008), http://dx.doi.org/10.1002/anie.v47:27.

7. M. E. Stewart et al., "Quantitative multispectral biosensing and 1D imaging using quasi-3D plasmonic crystals," Proc. Natl. Acad. Sci. U. S. A. 103(46), 17143-17148 (2006), http://dx .doi.org/10.1073/pnas.0606216103.

8. R. K. Gupta et al., "Enhancing charge-storage capacity of non-volatile memory devices using template-directed assembly of gold nanoparticles," Nanoscale 4(7), 2296-3000 (2012), http://dx.doi.org/10.1039/c2nr12134d.

9. D. K. Yun et al., "Fabrication of TiO 2 memristive arrays by Step and flash imprint lithography," J. Nanosci. Nanotechnol. 11(1), 696-700 (2011), http://dx.doi.org/10.1166/jnn.2011.3282.

10. B. Cui et al., "Fabrication of large area nanoprism arrays and their application for surface enhanced Raman spectroscopy,' Nanotechnology 19(14), 145302 (2008), http://dx.doi.org/ 10.1088/0957-4484/19/14/145302.

11. M. Singh et al., "Optical properties of d.c. magneto sputtered tantalum and titanium nanostructure thin film metal hydrides," Bull. Mater. Sci. 33(5), 569-573 (2010), http://dx.doi .org/10.1007/s12034-010-0087-8. 
12. C. J. Ting, C. F. Chen, and C. J. Hsu, "Heat-insulation film with gold layer deposited on antireflection subwavelength-structured surface," Optik 121(20), 1877-1880 (2010), http:// dx.doi.org/10.1016/j.ijleo.2009.05.013.

13. E. U. Kim et al., "Templated assembly of metal nanoparticles in nanoimprinted patterns for metal nanowire fabrication," Nanotechnology 20(35), 355302 (2009), http://dx.doi.org/10 .1088/0957-4484/20/35/355302.

14. G. Xia, S. Wang, and S.-J. Jeong, "A universal approach for template-directed assembly of ultrahigh density magnetic nanodot arrays," Nanotechnology 21(48), 485302 (2010), http:// dx.doi.org/10.1088/0957-4484/21/48/485302.

15. K.-M. Lim et al., "Development of metal etch mask by single layer lift-off for silicon nitride photonic crystals," Microelectron. Eng. 88(6), 994-998 (2011), http://dx.doi.org/10.1016/j .mee.2010.12.113.

16. F. De Angelis et al., "Emerging fabrication techniques for 3D nano-structuring in plasmonics and single molecule studies," Nanoscale 3(7), 2689-2696 (2011), http://dx.doi.org/10.1039/ c1nr10124b.

17. A. T. Hindmarch, D. E. Parkes, and A. W. Rushforth, "Fabrication of metallic magnetic nanostructures by argon ion milling using a reversed-polarity planar magnetron ion source," Vacuum 86(10), 1600-1604 (2012), http://dx.doi.org/10.1016/j.vacuum.2012.02.019.

18. S. Kim et al., "Nanoimprinted plasmonic nanocavity arrays," Opt. Exp. 21(13), 15081-15089 (2013), http://dx.doi.org/10.1364/OE.21.015081.

19. S. H. Jeon et al., "Ultraviolet nanoimprinted polymer nanostructure for organic light emitting diode application,” Appl. Phys. Lett. 92(22), 223307 (2008), http://dx.doi.org/ $10.1063 / 1.2939554$.

20. K. Ishihara et al., "Organic light-emitting diodes with photonic crystals on glass substrate fabricated by nanoimprint lithography," Appl. Phys. Lett. 90(11), 111114 (2007), http://dx .doi.org/10.1063/1.2713237.

21. H. H. Cho et al., "Solution-processed photonic crystals to enhance the light outcoupling efficiency of organic light-emitting diodes," Appl. Opt. 49(21), 4024-4028 (2010), http://dx.doi.org/10.1364/AO.49.004024.

22. H. H. Cho et al., "Planarization of nanopatterned substrates using solution process to enhance outcoupling efficiency of organic light-emitting diodes," Curr. Appl. Phys. 10(4), e139-e142 (2010), http://dx.doi.org/10.1016/j.cap.2010.08.027.

23. X. Zhang, B. Sun, and R. H. Friend, "Metallic photonic crystals based on solutionprocessible gold nanoparticles," Nano Lett. 6(4), 651-655 (2006), http://dx.doi.org/10 $.1021 / \mathrm{n} 10523610$.

24. B. Yang et al., "Electrochemical deposition of silver nanoparticle arrays with tunable density," Langmuir 25(1), 55-58 (2009), http://dx.doi.org/10.1021/la803559c.

25. W. Frey, C. K. Woods, and A. Chilkoti, "Ultraflat nanosphere lithography: a new method to fabricate flat nanostructures," Adv. Mater. 12(20), 1515-1519 (2000), http://dx.doi.org/10 .1002/(ISSN)1521-4095.

26. B. Jung and W. Frey, "Large-scale ultraflat nanopatterned surfaces without template residues," Nanotechnology 19(14), 145303 (2008), http://dx.doi.org/10.1088/0957-4484/19/14/145303.

27. P. Viste et al., "Enhancement and quenching regimes in metal-semiconductor hybrid optical nanosources," ACS Nano 4(2), 759-764 (2010), http://dx.doi.org/10.1021/nn901294d.

28. G. F. Walsh and L. D. Negro, "Engineering plasmon-enhanced Au light emission with planar arrays of nanoparticles," Nano Lett. 13(2), 786-792 (2013), http://dx.doi.org/10.1021/ nl304523v.

29. K. Ray et al., "Distance dependence of surface plasmon-coupled emission observed using Langmuir-Blodgett films,” Appl. Phys. Lett. 90(25), 251116 (2007), http://dx.doi.org/10 $.1063 / 1.2751125$.

30. Y. Wang et al., "Radiative rate enhancements in ensembles of hybrid metal-semiconductor nanostructures," Phys. Rev. Lett. 102(16), 163001 (2009), http://dx.doi.org/10.1103/ PhysRevLett.102.163001.

31. F. Yan and X. W. Sun, "A plasmonically enhanced charge generation layer for tandem organic light emitting device," Appl. Phys. Lett. 102(4), 043303 (2013), http://dx.doi .org/10.1063/1.4789979. 
32. A. Simo et al., "Formation mechanism of silver nanoparticles stabilized in glassy matrics," J. Amer. Chem. Soc. 134(45), 18824-18833 (2012), http://dx.doi.org/10.1021/ja309034n.

Jun-Hyuk Choi received his $\mathrm{PhD}$ in 2000 from the Department of Mechanical Engineering, University of California at Berkeley. He joined KIMM (Korea Institute of Machinery and Materials) in Daejeon, South Korea, in 2003. At present, he is a principle researcher in KIMM. Also, he is an associate editor in International Journal of Precision Engineering and Manufacturing and Trans. Kor. Soc. Mech. Eng. C. His research interests include nanoimprint, metal nanopatterning and nanostructures-based photonic, and plasmonic applications.

Sang-Keun Sung received his MS degree in 2011 from the department of physics in Chungnam National University, Daejeon, South Korea. He joined in the department of nanomanufacturing technology of KIMM (Korea Institute of Machinery and Materials) in 2011, and is presently an assistant researcher in KIMM. His research interests include nanopatterning using nanoimprint and its applications to plasmonic optoelectronics.

Chul-Hyun Kim received his MS in 2011 from the department of display engineering, Busan National University, South Korea. He majored in the materials and fabrication for organic light emitting devices. He was an assistant researcher in KIMM (Korea Institute of Machinery and Materials) in Daejeon, South Korea, from 2011 to 2013. Then he worked in hybrid nanoimprint and its application to plasmonic OLED. He joined LG chemicals in 2013. Now, he is a staff engineer in the division of organic lighting.

Yeon-Ho Jung received his BS degree in 2012 from the Department of Mathematics in Korea University, South Korea. He majored in both mathematics and industrial engineering. He is presently enrolled in the University of Science and Technology for his graduate program. Coupled with this graduate research program, he is affiliated with KIMM (Korea Institute of Machinery and Materials). His research areas include nanopatterning using nanoimprint and nanostructuredriven color imaging.

Joo-Yun Jung received his $\mathrm{PhD}$ in 2010 from the department of electrical and computer engineering, University of Texas at Austin. He joined KIMM (Korea Institute of Machinery and Materials) in Daejeon, South Korea, in 2010. At present, he is a senior researcher in KIMM. His research interests include nanoimprint, metamaterials and nanostructures-based photonic, and plasmonic applications.

Jun-Ho Jeong received his undergraduate training at Hanyang University (BS 1990) and his MS at KAIST where he completed his $\mathrm{PhD}$ in mechanical engineering in 1998. He is currently a principle search scientist at KIMM. His research interests include nanofabrication, especially nanoimprint lithography (NIL) and nanofluidics.

Eung-Sug Lee is presently a vice president of KIMM as a tenured principle researcher. He used to serve as a director of the Division of Nano and Convergence Technology in the National Research Foundation of Korea from 2009 to 2011. He has been credited with more than seventy patents, and a contributing author of thirties SCI publications during the last 5 years in the field of nanoimprint, plasmonic nanostructure, and various nanopattern-based optoelectronics. 